\title{
Possible Application of the System Loss Concept at ELF ${ }^{1}$
}

\author{
Kenneth A. Norton
}

(February 25, 1960)

\begin{abstract}
A brief description is given of the possible application of the system loss concept at extremely low frequencies. A method for allowing for the effect of external noise levels is outlined.

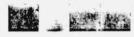

The system loss concept [1] ${ }^{2}$ has been recommended $[2,3]$ by the Consultative Committee on International Radio as a precise means for describing the overall performance of a radio system including not only the effects of the intervening propagation medium, but also the gains and losses in the transmitting and receiving antennas. The purpose of this brief paper is to call this concept to the attention of engineers designing radio systems which are expected to operate in the extremely low frequency (ELF) or lower frequency ranges. The efficient design of systems operating at such low frequencies is especially difficult, and it is believed that the use of the system loss concept may thus prove to be quite helpful.

Any radio system may be usefully described [4] by means of the simple T network of figure 1 . The accessible terminals of the transmitting antenna $a$ and of the receiving antenna $b$ are denoted by $A A$ and $B B$, respectively. The central member of the $T$ is the mutual impedance $z_{m} \equiv\left|z_{m}\right| \exp (i \phi)$ between the two antennas, and this contains implicitly all of the effects of the propagation. The self-impedances of the two antennas are $z_{a}$ and $z_{b}$; thus the impedance at the terminals $A A$, with $B B$ open-circuited, is $z_{a} \equiv r_{a}^{\prime}+i x_{a}$, while the impedance at the terminals $B B$, with $A A$ open-circuited, is $z_{b} \equiv r_{b}^{\prime}+i x_{b}$.

${ }^{1}$ Contribution from Central Radio Propagation Laboratory, National Bureau of Standards, Boulder, Colo.; paper presented at Conference on the Propagation of ELF Radio Waves, Boulder, Colo., Jan. 25, 1960.

2 Figures in brackets indicate the literature references at the end of this paper.

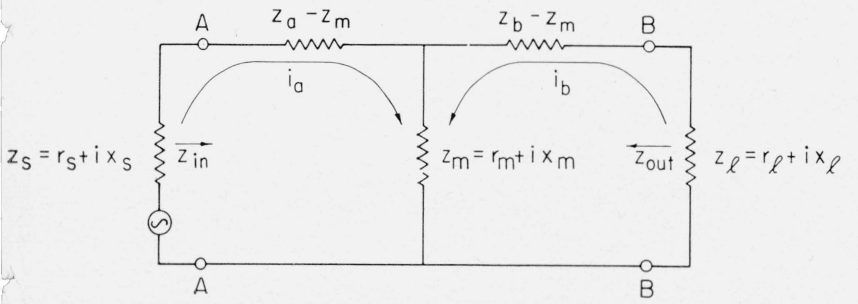

FIGURE 1. T-network for system loss analysis.
The system loss is defined as the dimensionless ratio, $p_{t} / p_{a}$, where $p_{t}$ is the radiofrequency power input to the terminals of the transmitting antenna and $p_{a}$ is the resultant radiofrequency signal power available at the terminals of the receiving antenna. The system loss is usually expressed in decibels.

$$
L_{s}=10 \log _{10}\left(p_{t} / p_{a}\right)=P_{t}-P_{a} .
$$

In the design of the system it will normally be desirable to minimize $\bar{L}_{s}$. It was shown [1] that this may be accomplished by simultaneously matching the source impedance $z_{s}$ to the complex conjugate of $z_{\text {in }}$ and the load impedance $z_{l} \equiv r_{l}+i x_{l}$ to the complex conjugate of $z_{\text {out }}$. For this condition of matching

$$
L_{s}\left(z_{s}=z_{\text {in }}^{*} ; z_{l}=z_{\text {out }}^{*}\right)=10 \log _{10}\left[\frac{2 r_{a}^{\prime}\left(r_{b}^{\prime}+r_{l}\right)}{\left|z_{m}^{2}\right|}-\cos (2 \phi)\right]
$$

where

$$
\begin{gathered}
z_{l}=r_{b}^{\prime}\left\{1-\frac{\left|z_{m}^{2}\right| \cos (2 \phi)}{r_{a}^{\prime} r_{b}^{\prime}}-\frac{\left|z_{m}^{4}\right| \sin ^{2}(2 \phi)}{4 r_{a}^{\prime 2} r_{b}^{\prime 2}}\right\}^{\frac{1}{2}} \\
-i x_{b}+i r_{b}^{\prime}\left\{\frac{\left|z_{m}^{2}\right| \sin (2 \phi)}{2 r_{a}^{\prime} r_{b}^{\prime}}\right\} .
\end{gathered}
$$

At low frequencies the dominant component of the available noise power $p_{a n}$ appearing across the terminals $B B$ will usually have been received after propagation from a noise source with power $p_{n}$ over propagation paths with an effective system loss $L_{n}=10 \log _{10}\left(p_{n} / p_{a n}\right)$. In this case we see by the following equation that a maximum signal-to-noise ratio may be obtained by minimizing $\left(L_{s}-L_{n}\right)$.

$$
P_{a}-P_{a n}=P_{t}-P_{n}-\left(L_{s}-L_{n}\right) .
$$

In minimizing $\left(L_{s}-L_{n}\right)$ it is important to notice that most changes in the receiving system will affect $L_{s}$ and $L_{n}$ alike and will thus not improve the signal-tonoise ratio to the extent that the dominant component of the noise is not in the receiving circuits themselves. 
It may be helpful to the reader to list some of the advantages and disadvantages of the use of the system loss concept. Its principal advantages are that it: (1) Makes possible a single, precise measurement of the entire system performance; (2) shows explicitly the influence of such circuit parameters as antenna impedances; and (3) combines in a single parameter the interdependent effects of the propagation and the antenna gains and losses.

The principal disadvantage of its use arises from the fact that it does combine the influence of at least partially independent factors. Thus, it is certainly a great advantage to be able to study the transmitting antenna, the propagation medium, and the receiving antenna independently. In those cases where these factors cannot be considered to be independent, the system loss concept must be used for a precise description of the system.

\section{References}

[1] K. A. Norton, System loss in radio wave propagation, J. Research NBS, 63D, 53 (1959).

[2] Documents of the IXth Plenary Assembly of the C.C.I.R., Los Angeles, Calif., Vol. I, Recommendation No. 241, pp. 128 to 130 (1959); and Vol. III, Rept. No. 112, pp. 94 to 99, Intern. Telecommun. Union, Geneva, Switzerland (1959).

[3] K. A. Norton, System loss in radio wave propagation, Proc. IRE 4y, 1661 (1959).

[4] James R. Wait, Transmission of power in radio propagation, Electron. and Radio Engr. 36, 146 (1959).

(Paper 64D4-75) 